\title{
An algorithm for constructing a truss network with the modular truss-Z system for pedestrian traffic in a 3D environment
}

\author{
M. Zawidzki \\ Ritsumeikan University, Japan
}

\begin{abstract}
An algorithm for creating a truss network with the truss- $Z$ system in a $3 D$ environment with obstacles is demonstrated. This modular truss system is designed for pedestrian traffic and connects any given number of points in space (terminals), allowing "branching" and "looping". The concept of the truss-Z is based on a universal module that is subject to geometrical transformations, allowing the construction of a structural link along virtually any 3D path. The emerging form of the structure has organic aesthetic qualities. The elements of the environment model real obstacles, such as roads, buildings and watercourses, which may constrain both the run of the truss and the placement of the supports. An example of a truss- $Z$ network connecting six terminals in an environment with three solids is shown.
\end{abstract}

Keywords: modular, truss system, 3D, organic, skyway, emergence.

\section{Introduction}

The purpose of this project is to develop a construction system for pedestrian walkways that is extremely flexible and inexpensive [2].

Flexibility: the system can realize a large number of geometrical tasks and one segment of the truss system can link efficiently any two terminals in $3 \mathrm{D}$ space. The system also supports branching of segments and (closed) loops.

Inexpensiveness: the system is modular with the run of the truss composed of only two modular elements. The supporting system is based on a single member. Elements are meant to be prefabricated in large numbers and assembled on site (preferably without the necessity for heavy equipment) or can be made on site using templates and locally available materials (for example timber in a forest). 
The system obviously must be sound and safe. The same concept can be adjusted in scale and shape for different purposes such as cycle paths or other transportation tasks.

There are many examples of very complicated geometries realized in construction [3]. Almost always, the complexity comes at the expense of modularity with nearly every member being custom-made. On the other hand, there are a variety of construction modular systems, but then high modularity produces rather simple forms with very limited geometries $[4,5]$.

The most important aspect of the truss- $Z$ is that it is the first structural system which gives almost limitless geometrical possibilities for creating 3D paths and at the same time is modular. Unlike other modular systems, the unit module is not overly simplified. Within the truss-Z, simple forms are constructed by nontrivial assembly of fairly complex elements, as opposed to the usual modular systems, where a simple form is achieved by assembly of simple units. As already mentioned, the latter approach results in very limited geometrical possibilities within a system. The core of this innovative system is the idea that no shape or direction is preferred, for example following a straight line is merely one of a great many possible paths.

There are also a number of, (mostly theoretical) projects (usually realized at most as scale models), claiming modularity but in a topological sense, where in fact each "module" has a different geometry [6]. Nonetheless, the concept of modularity represented in this paper refers to the geometrical modularity, that is the geometrical identity of the units.

\section{The module}

The truss- $Z$ invented by the author as a structural spatial truss system was originally designed for pedestrian traffic and therefore has specific geometrical properties. Practically, people perform any kind of movement on the horizontal surface, while the vertical one is very limited and troublesome for both the user and the designer. This fact is reflected in many building and construction regulations by limiting the slope to approximately 1:10 rise to run ratio (depending on the purpose and the local law) [7]. The vertical angle of the module of the truss- $Z$ system varies from $8.05^{\circ}$ at the longer side, to $11.31^{\circ}$ at the axis, to $27.80^{\circ}$ at the short side. On the other hand, the possibility of tight turns in the horizontal plane is desirable and allows the structure to change direction and meander around obstacles. The horizontal angle of a unit is $30^{\circ}$, so by using only three units a right angle turn can be completed at a relatively very small radius. The dimensions are a compromise between many constraints and requirements and as a result the module supports a number of configurations including a vertical spiral. The slope can be further reduced, but then the ability to create a spiral may be limited.

The conceptual purity of this system is a result of the fact that all the geometrical tasks of the main structure, that is connecting given terminals, are realized by using a single element subject to geometrical transformations: 
reflection, rotation, translation, (and in a certain sense- scale). Although the system works with uniform modules, the modules can be of any size [8].

In the virtual world of the computer program, there is only one unit (R) used and transformed, but since in the physical space, objects cannot be subject to symmetry transformation, for a real truss, a reflected (symmetrical) unit has to be produced as well. Further in the text, both "right" and "left" units are considered independently.

The units are named according to the right-hand-rule; the basic module is called " $R$ " (right), since it turns left and goes up. When this unit is rotated along the vertical axis by $150^{\circ}$ it becomes an "R2" (rotated right) unit. The mirror refection of the "R" unit by a vertical plane makes the "L" (left) unit and the rotation of the "L" unit is an "L2" (rotated left) unit. All these variations have different "vectors" equivalent to the change of the position in space: "up and left" (R), "up and right" (L), "down and left" (R2) and "down and right" (L2) as shown in Figure 1. The module is a rigid spatial frame-truss hybrid.

Although the vertical angle of a unit at the centre is $11.31^{\circ}$, it is also possible to construct a truss with lower slopes or completely flat. For example a sequence of units $\{\mathrm{R}, \mathrm{R} 2, \mathrm{R}, \mathrm{R} 2, \mathrm{R}, \mathrm{L}\}$ has the vertical angle $3.8^{\circ}$ and complies even with wheelchair ramp regulations (ratio between the rise and the length of the run $1: 12=4.8^{\circ}$ of the vertical angle) [9]. Examples of sequences of the units and the resulting structures are shown in Figure 2. The colouring convention is R-green, R2- cyan, L- red, L2- yellow.

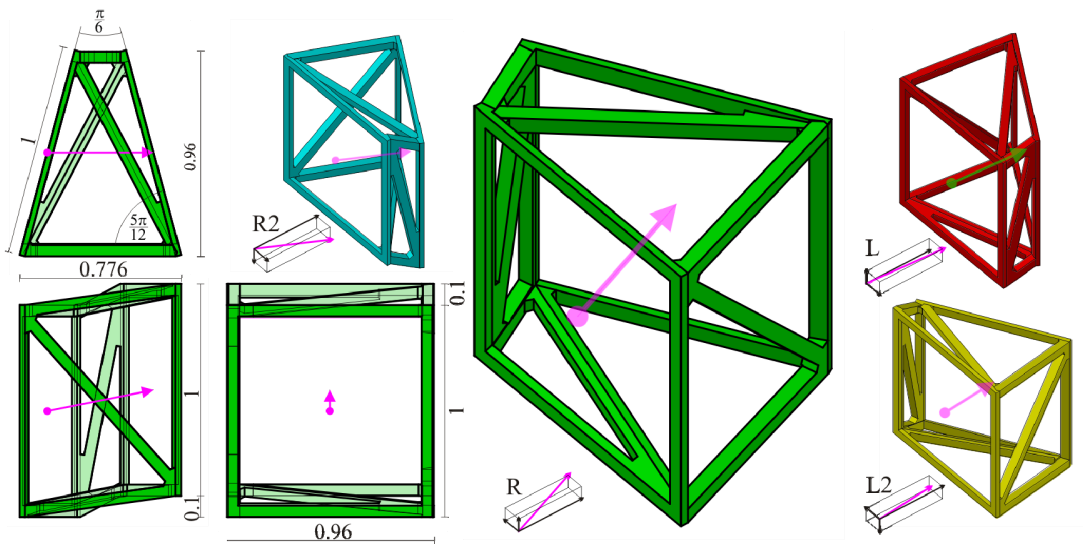

Figure 1: The geometrical properties of the module and all its variations: $\mathrm{R}$, R2, L, L2. 


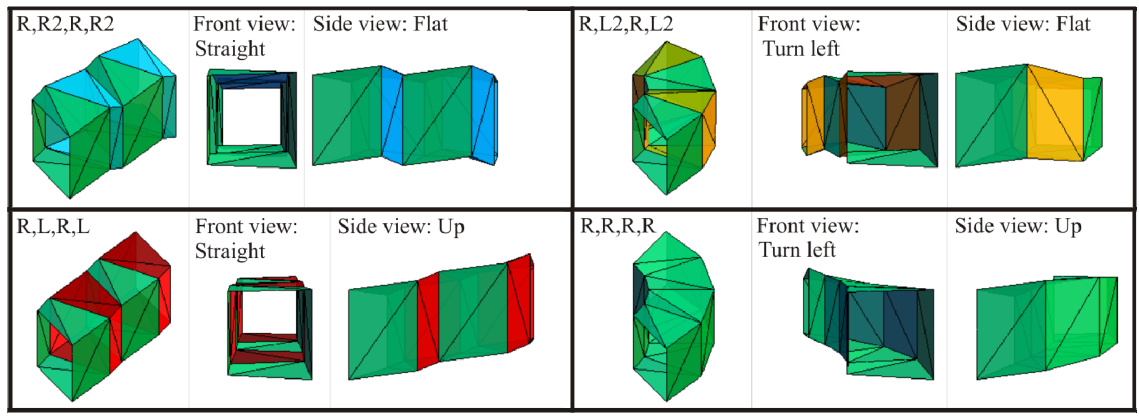

Figure 2: $\quad$ Examples of sequences of units and the resulting spatial structures.

\section{Creating the path for the truss}

For creating the runs of the truss the following assumptions have been made:

1) The truss must connect any given number of terminals (points in space).

2) The segments of the truss must not collide with any objects: obstacles and other segments of the truss.

3) The truss should be optimal so the number of modules used for the structure to be minimum.

4) The network of connections should be as simple as possible by minimizing the number of additional junction units (tangent points).

If the number of given points to be connected is greater than two, it is necessary to introduce a junction as shown in Figure 3. There are two possible such twin-unit configurations: $\{\mathrm{R}, \mathrm{L}\}$ and their rotation: $\{\mathrm{R} 2, \mathrm{~L} 2\}$. From such a unit three (node degree $=3$ ) or four (node degree $=4$ ) branches can be constructed.

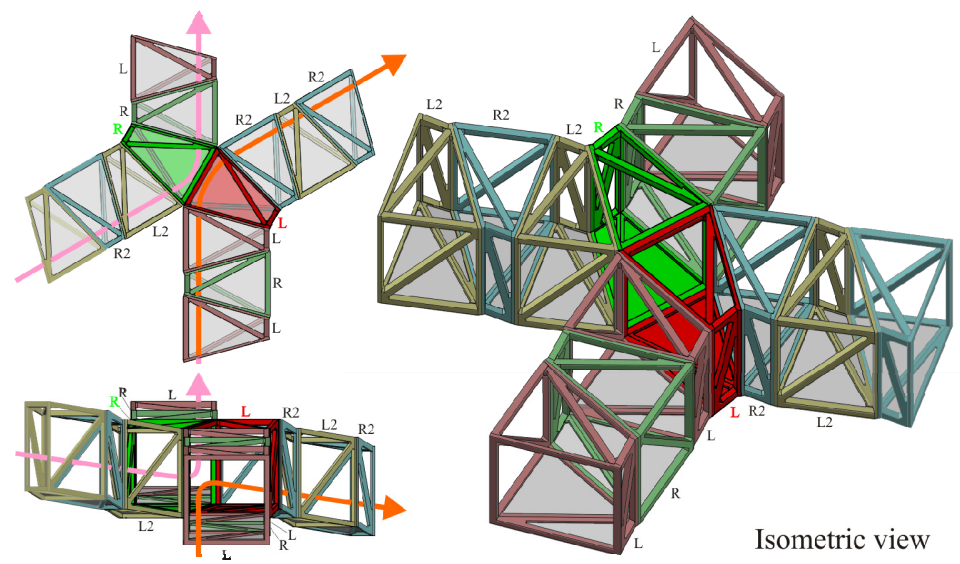

Figure 3: The junction unit (with four branches) placed at the tangent point of two paths. 


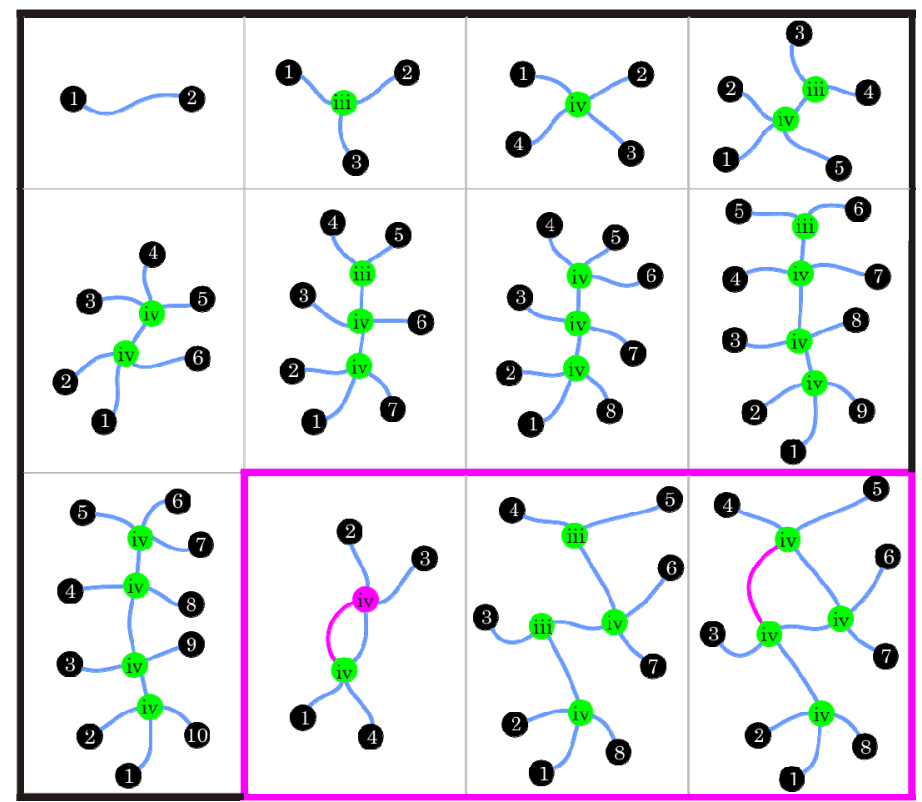

Figure 4: The smallest path networks for a given number of terminals (shown in black from two to ten). The junction units are shown in green (colour online only - the numbers indicate the degree of the node). The three schemes on the bottom right show examples with redundant junction units.

Although number of junction units is to be minimized, there may be conditions requiring the introduction of redundant junction units (Figure 4).

The minimum number of junction units $\left(\mathrm{n}_{\mathrm{j}}\right)$ depends on the number of terminals $\left(\mathrm{n}_{\mathrm{t}}\right)$ and can be expressed as follows:

$$
\mathrm{n}_{\mathrm{j}}=\left(\mathrm{n}_{\mathrm{t}}+\operatorname{Mod}\left[\mathrm{n}_{\mathrm{t}}, 2\right]\right) / 2-1 \text {, }
$$

\section{The general algorithm}

Constructing the truss network connecting a given number of terminals in a constrained 3D environment (obstacles) consists of the following steps:

1) Input data- positions of terminals and obstacles in plan (Figure 5.1). The terminals are numbered accordingly and the minimal number of junctions is calculated.

2) Triangulation of the plan and computing the positions of the centroids of the triangles (Figure 5.2).

3) Generating subsets of nodes (Figure 5.3). 
4) Finding (possibly the shortest) segmented path- minimum spanning tree (MST) of the subsets of the nodes on the grid constructed from the centroids of the triangles (Figure 5.4) so that:

4.1) none of the segments crosses other segment.

4.2) none of the segments crosses an obstacle.

4.3) the sum of the lengths of all the segments is not greater than a certain value.

If it is not possible- additional junction unit is introduced and the procedure runs from step 3 .

5) Constructing the splines of the guiding paths from the segmented lines so that the minimal curvature is preserved due to the geometric characteristics of the truss- $Z$ modules (Figure 5.5).

6) Generating the truss structure along the paths (Figure 5.6).

7) Optimization of the truss network by minimizing the overall number of modules for the structure using a heuristic method such as a genetic algorithm.

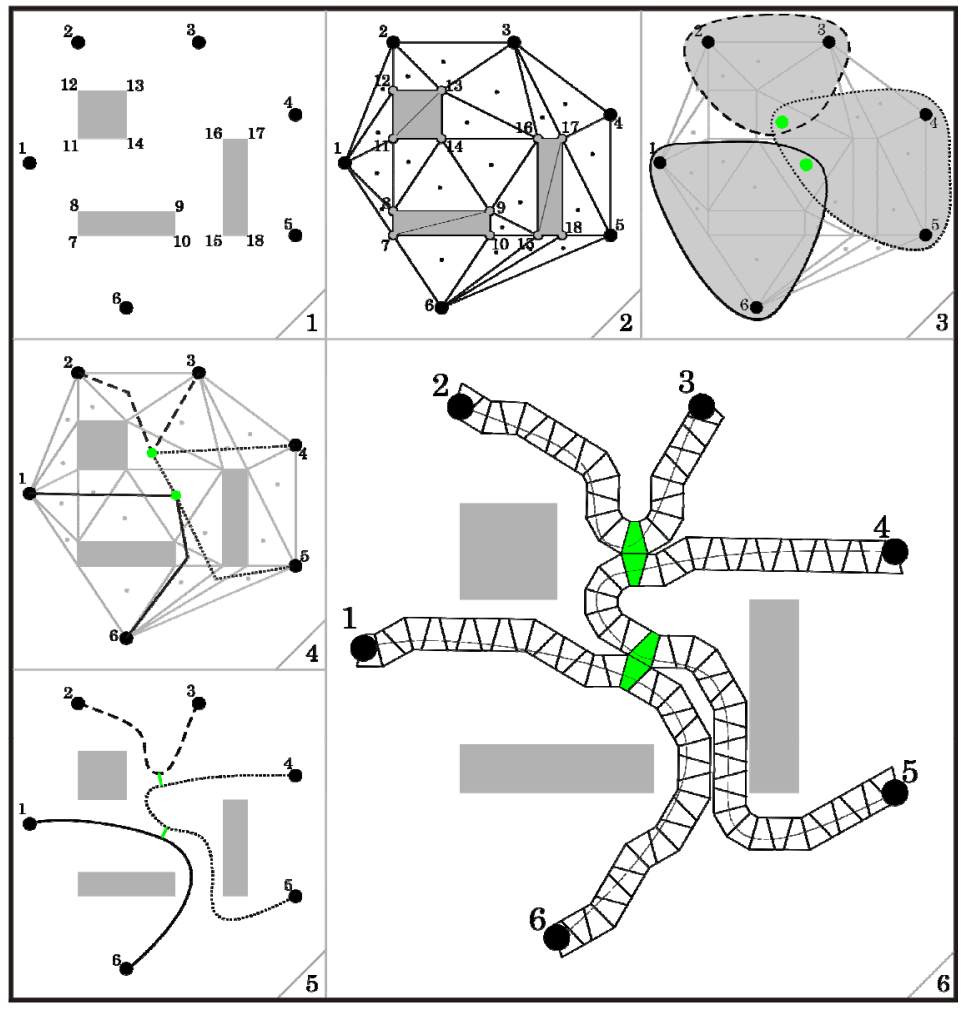

Figure 5: Schematic visualization of the general algorithm. 


\section{The modules alignment along a path}

The run of a truss follows a guide path, which can be given as any parametric function. In this example splines were applied, since they are an intuitive way of modelling transportation paths. In this discrete optimization, one of four possible units (two modules and two rotations) which gives the minimum value of the objective function is chosen. At each step, the list of two values related to the given guide path is calculated for all four possible configurations (Figure 6):

1) The minimum distance (d) between the centroid of the module (C) and the guide path (p).

2) The normalized dot product of the direction of the curve (the guide path) $\left(r^{\prime}\right)$ at the closest point to the centroid of the module and the vector (v) of the unit (p).

$$
\text { Minimize[u*d/b+(1-u) }(1-|v \bullet r '[p]|)] \text {, where }
$$

$d$ is the smallest distance between the centroid $(C)$ of a unit and the curve $(p)$; $v$ is the vector of the unit;

$r^{\prime}[p]$ is the direction of the guide path in point $p$; $u$ and $b$ are parameters described below.

The parameter $u$ is a weight ranging from 0 to 1 and moves the influence from the normalized dot product of the direction of the curve and the vector of the unit to the distance between the centroid of a unit and the curve. Since the objective function depends both on distance and angle which cannot be normalized, the parameter $b$ adjusts the ratio between them. The values of parameters $u$ and $b$ were optimized so the number of units to follow a given path was minimal (Figure 7).
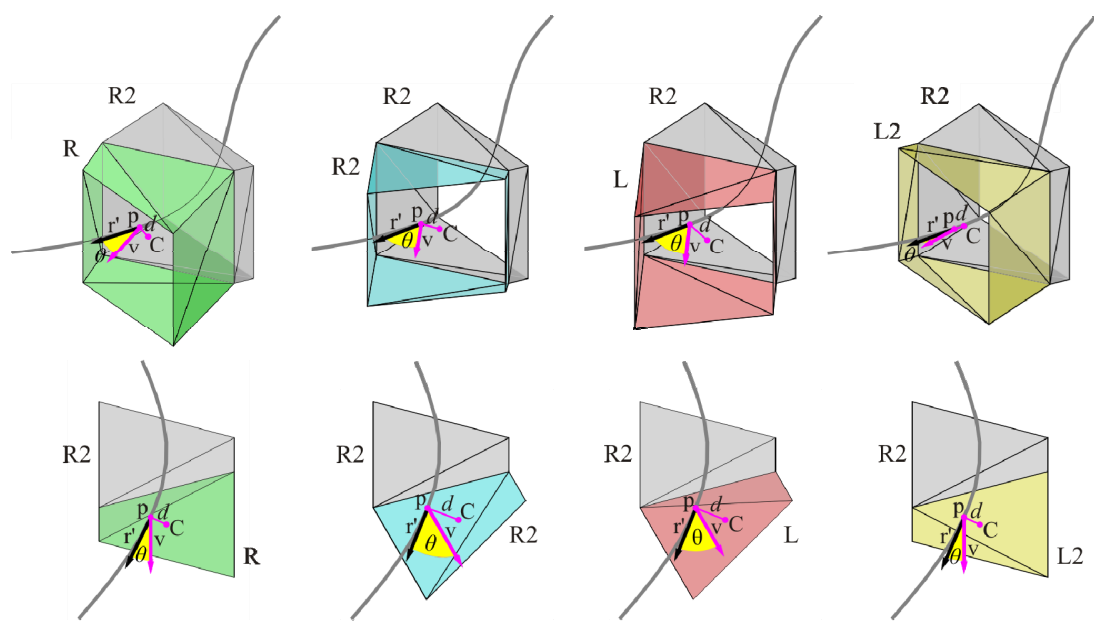

Figure 6: The four possible configurations for the "next" unit. The top row: isometric views, the bottom row: top views. 
The algorithm operates on paths and list of sub-paths and consists of the following steps:

1) All the segments of the truss containing two junction points are constructed (Figure 8.1).

2) The junction units are completed by attaching the "mirrored" units (Figure 8.2).

3) These units become starting points for two half-runs of the remaining two paths. (Figure 8.2).
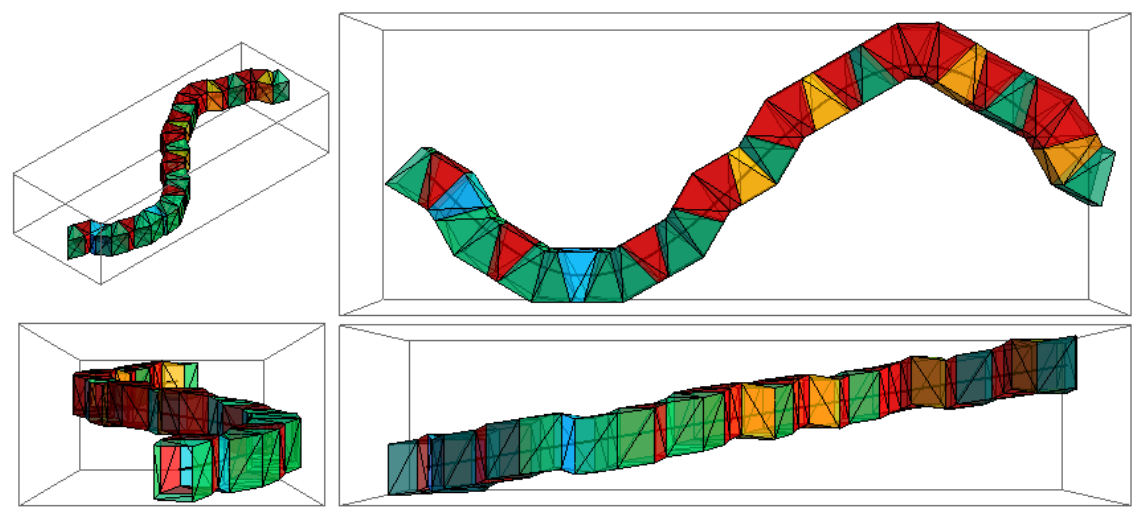

Figure 7: $\quad u=0.5, b=100$; the balance between maintaining a small distance to the guide path and following its curvature. The number of units to follow the given path is 33 (minimum). The list of units: $\{\mathrm{R}, \mathrm{L}, \mathrm{R} 2, \mathrm{R}, \mathrm{R}, \mathrm{L}, \mathrm{R}, \mathrm{R}, \mathrm{R} 2, \mathrm{R}, \mathrm{R}, \mathrm{L}, \mathrm{R}, \mathrm{R}, \mathrm{L}, \mathrm{L}, \mathrm{L} 2, \mathrm{R}, \mathrm{L}, \mathrm{L}, \mathrm{L} 2, \mathrm{~L}, \mathrm{R}, \mathrm{L}, \mathrm{L}, \mathrm{L}, \mathrm{L} 2$, $\mathrm{L}, \mathrm{R}, \mathrm{L}, \mathrm{L}, \mathrm{L} 2, \mathrm{R}\}$.
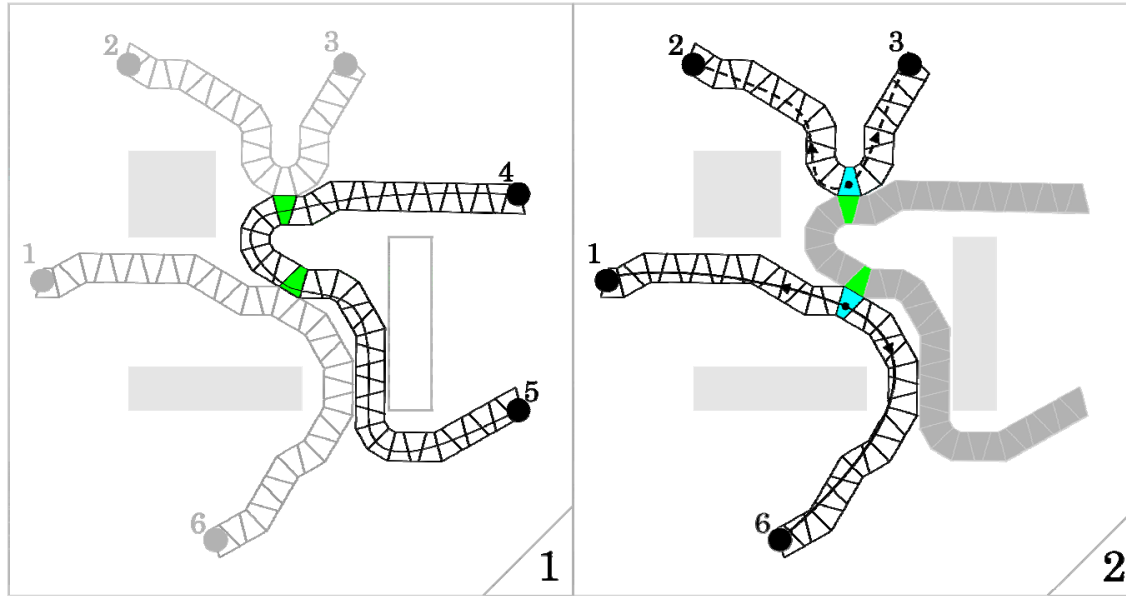

Figure 8: The sequence of constructing the segments of the truss network. 


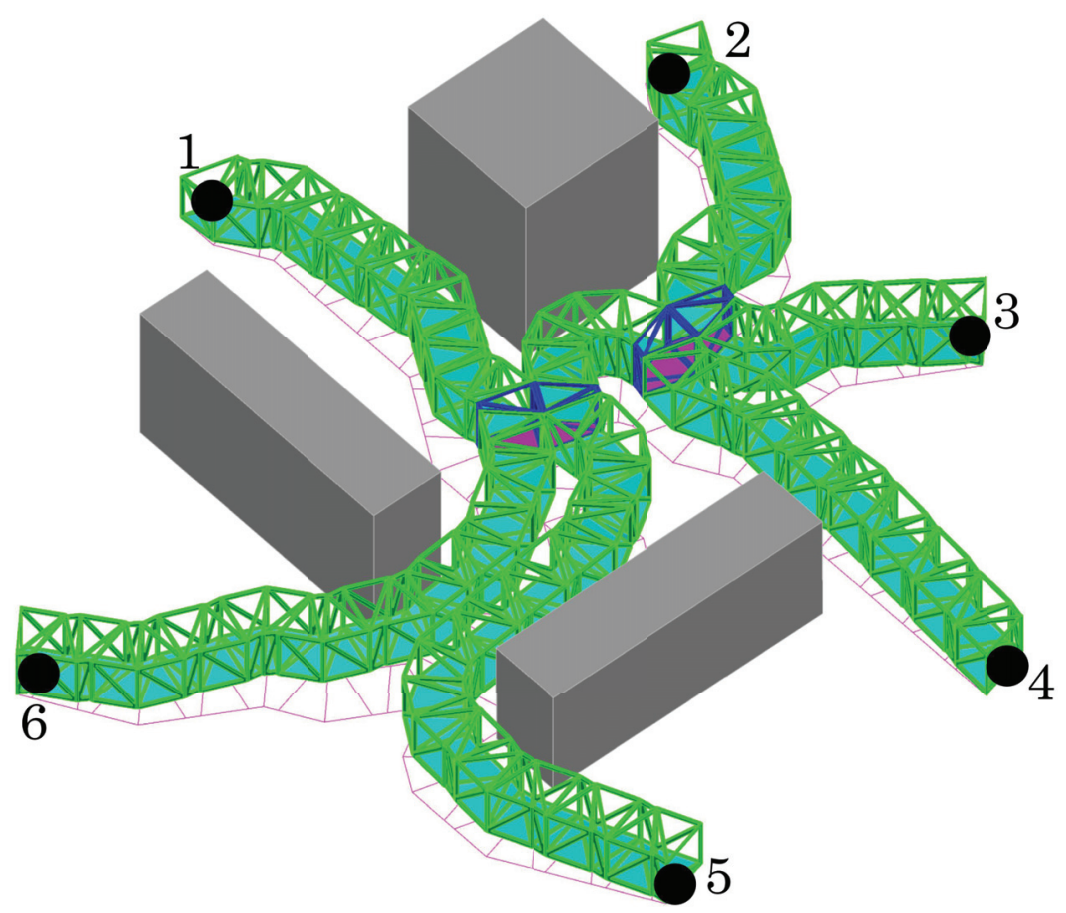

Figure 9: 3D computer model of the completed truss network for given terminals and obstacles (the supports are not shown).

The splines of the guide paths are three dimensional and the nodes in the centre were elevated to simulate an overpass. The main runs of the completed truss network are shown in Figure 9.

\section{The supporting structure}

Besides the global stability, which is a separate problem, the structure is rigid due to the rigidity of the modules. Nevertheless, due to the finite strength of real materials, it is necessary to introduce a support system which is constructed from spatially rigid units of uniform length members (regular tetrahedra). Usually in order to avoid buckling, the supporting structure is constructed so that the load is carried in the shortest way to the foundation. The proposed geometry of the supporting system based on tetrahedron may seem less intuitive, but with the use of a single unit, it allows the build of supporting elements in any direction (within certain accuracy) and even permits "going around" obstacles. Although, this is not a biomimetic project since it does not mimic any biological forms or mechanisms, the resemblance to natural processes is worth mentioning. Like many naturally occurring structures, the same element (agent or unit in this case) is simple and at the same time universal enough to be used in a variety of ways [10]. 
The construction of the supporting structure is based on the algorithm for the single path segments of the truss. All except the first two elements of the support are made of regular tetrahedra with the length $a$ as shown in Figure 9. First, at the selected truss unit a (non-regular) tetrahedron is constructed with the three points of the truss unit as the base. Newly added elements have always length $a$. In general the third tetrahedron is already a regular one and so are all the following ones. Alike the main truss, it is constructed along a given guide path. The algorithm has analog parameters $u$ and $b$ and chooses one of three possible orientations of the next tetrahedron.

The selection of the points for the support is based on the following rules:

1) The maximum strain: The maximum span (number of unsupported modules) is limited to a given value (Figure 11.1).

2) Structural stability: the supports to be placed maximizing the balance of the truss (Figure 11.2).

3) Maximum eccentricity is limited to a given value (Figure 11.3).

An example of a complete truss network with supports in an urban environment is shown in Figure 12.

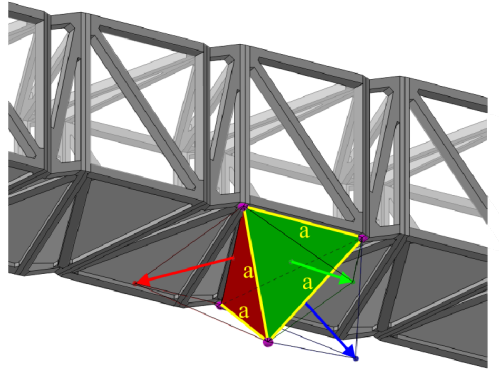

A bottom view of the first tetrahedron

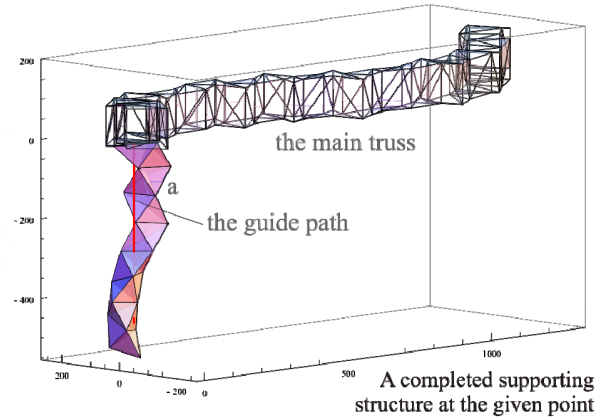

structure at the given point

Figure 10: The supporting structure. There are three possible directions in which the next tetrahedron can be added (red, green and blue arrows - colour online only) at every step.

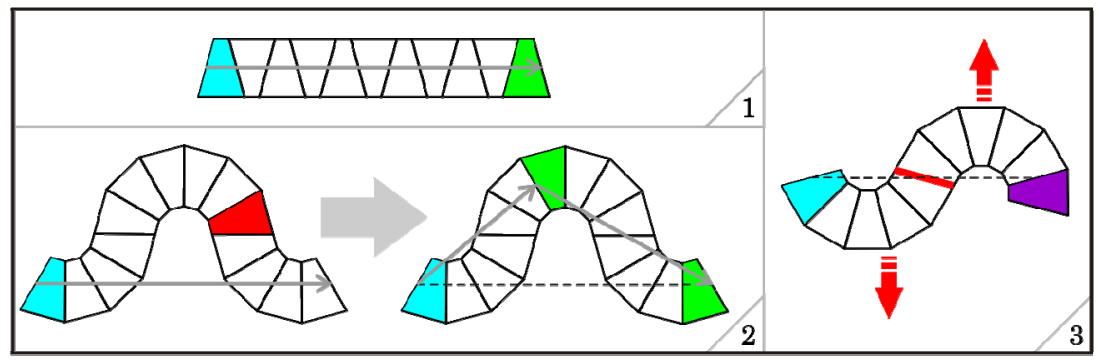

Figure 11: The placement of the supports: 1) the maximum span constraint, 2) stability, 3) eccentricity (the connection of maximum strain is indicated with red - colour online only). 


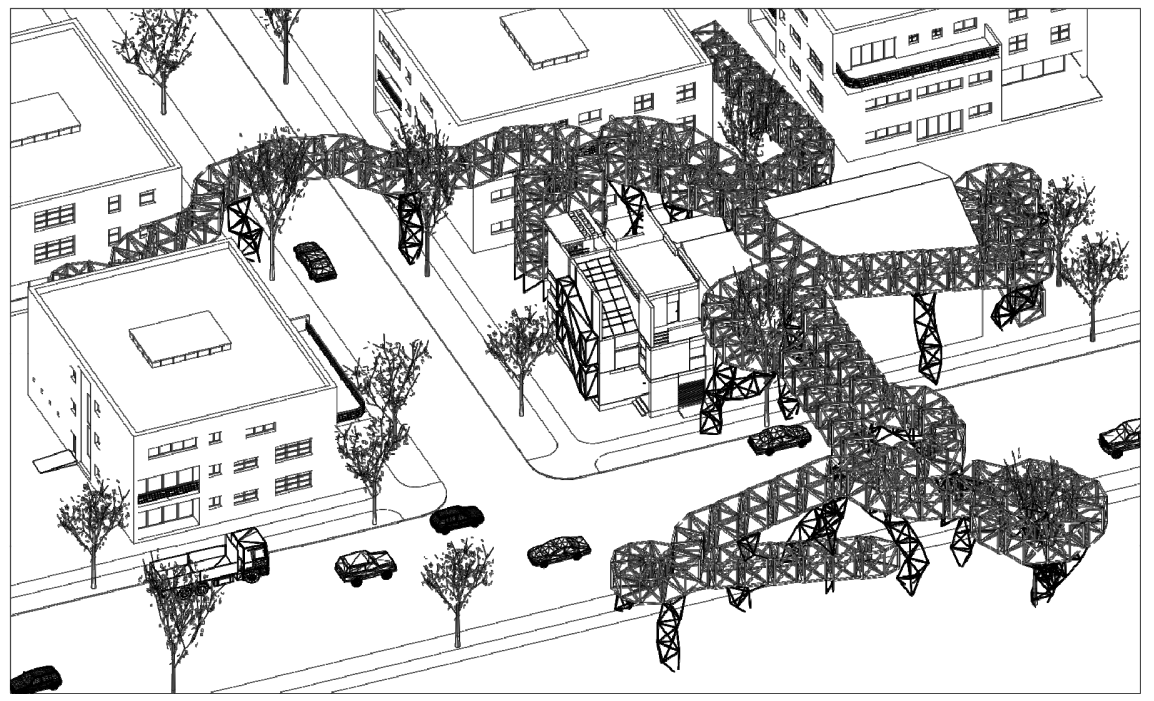

Figure 12: A computer model showing the complete truss network with supports, spiral and a variety of linkages in an urban environment.

\section{Conclusions}

1) An example of constructing a truss network with truss- $Z$ system for pedestrian traffic at given conditions has been demonstrated.

2) The system can be locally modified (without influencing the remainder of the structure) and adapted to changing conditions, for example by redirecting or increasing the capacity of the pedestrian traffic according to the time of the day [11].

3) Development of a joint system for on-site assembly is under consideration.

4) Although optimization methods were implemented in the algorithm shown above (MST, discrete optimization), the obtained solution is not globally optimal (for example minimizing the number of modules used). Application of heuristic method, a self-adaptive ant colony optimization (ACO) is under consideration.

\section{Acknowledgements}

I would like to cordially thank Professor Kazuyoshi Tateyama and Professor Ikuko Nishikawa from Ritsumeikan University for the support and encouragement. From Wolfram Research Inc., many thanks are due to Maxim Rytin for his tremendous help in the development of the algorithms and to Chris 
Hill for his contribution in the Graphic User Interface of the computer implementation.

\section{References}

[1] Bar-Cohen Y., Biomimetics Biologically Inspired Technologies, Taylor \& Francis Group (2006), p. 230.

[2] Luchsinger R.H., Crettol R., Plagianakos T.S., Temporary structures with Tensairity®; International Symposium IASS-SLITE - Acapulco (2008)

[3] Spuybroek L., NOX, Thames \& Hudson, (2004). p. 142-157

[4] “Allround Modular Truss System" by Wilhelm Layher GmbH \& Co. KG www.layher.co.nz/scaffold-project-allround-modular-truss-system.html

[5] Modular truss systems: (US Patents 5964068, 5826384, 7347030, 5390463)

[6] Spuybroek L., The architecture of variation, Thames \& Hudson, (2009). p. 127-129

[7] Bowman R., An introductory guide to the slip resistance of pedestrian surface materials, Strathfield, NSW: Standards Australia; Wellington: Standards New Zealand (1999).

[8] Staib G., Dörrhöfer A., Rosenthal M., Components and Systems: Modular Construction Design, Structure, New Technologies (2008).

[9] Billington, M. J., Bright, K. T., Waters J. R., The Building Regulations: Explained and Illustrated (2007).

[10] Jeronimidis, G., Bioinspiration for Engineering and Architecture: MaterialsStructures- Function, Proceedings of the $28^{\text {th }}$ Annual Conference of the ACADIA, Minneapolis (2008).

[11] Luchsinger R.H., Crettol R., Adaptable Tensairity, IASS International Conference on Adaptability in Design and Construction, Eindhoven (2006). 